\title{
Verwendung kinematischer Ketten bei der Generierung von Finite-Elemente-Modellen
}

\author{
DR.-ING. MARTIN WEBHOFER \\ TECHNISCHE UNIVERSITÄT MÜNCHEN, LEHRSTUHL FÜR FÖRDERTECHNIK MATERIALFLUSS LOGISTIK
}

\begin{abstract}
Vorliegende Arbeit stellt eine am Lehrstuhl für Fördertechnik Materialfluss Logistik der Technischen Universität München entwickelte Modellierungsmethode vor, die eine Abbildung von FiniteElemente-Modellen als kinematische Strukturen ermöglicht. Dabei besteht das Gesamtmodell aus einer Reihe einzelner FE-Teilmodelle, die durch Gelenke miteinander verbunden sind. Durch das Verändern weniger Parameter kann die räumliche Anordnung der Teilmodelle zueinander verändert werden. Somit lassen sich die unterschiedlichen Betriebszustände der realen Maschine durch ein einziges Modell abbilden, wodurch der Aufwand für die Modellierung, Berechnung und Auswertung derartiger Systeme erheblich reduziert werden kann.

This article describes a modelling method which was developed at the Institute of Material Handling, Material Flow, Logistics at the Technical University of Munich. This method allows for the representation of finite element models in the form of kinematic structures. The complete model consists of a series of single FE sub-models which are connected by links. By changing just a few parameters the spatial arrangement of these sub-models can be altered and the different operating states of real machines can be represented by a single model. Thanks to this the expense for modelling, calculating and evaluating such systems can be reduced considerably.
\end{abstract}

\section{Problemstellung und Zielsetzung}

Die Modellierung, Berechnung und Auswertung von Finite-Elemente-Strukturen, die je nach Betriebszustand unterschiedliche räumliche Ausprägungen haben, ist mit den konventionellen Modellierungsmethoden mit einem hohen Zeit- und Kostenaufwand verbunden. Am Beispiel der Gittermast-Fahrzeugkranberechnung lässt sich dieser Sachverhalt sehr gut zeigen.

Fahrzeugkrane mit Gittermastauslegern werden für einen breiten Bereich bezüglich Hubhöhe, Ausladung und Hublast eingesetzt. Lange Haupt- und Hilfsausleger erlauben Hubaufgaben über hohe Bauwerke hinweg. Die größten Lastmomente erreicht ein Fahrzeugkran mit kurzem Hauptausleger, Abspannmast und einem großen Superlift-Gegengewicht (vgl. Abb. 1). Um diesen, von der jeweiligen Hubaufgabe abhängigen, unterschiedlichen Anforderungen Rechnung zu tragen, ist ein derartiger Kran nach dem Baukastenprinzip konzipiert. 


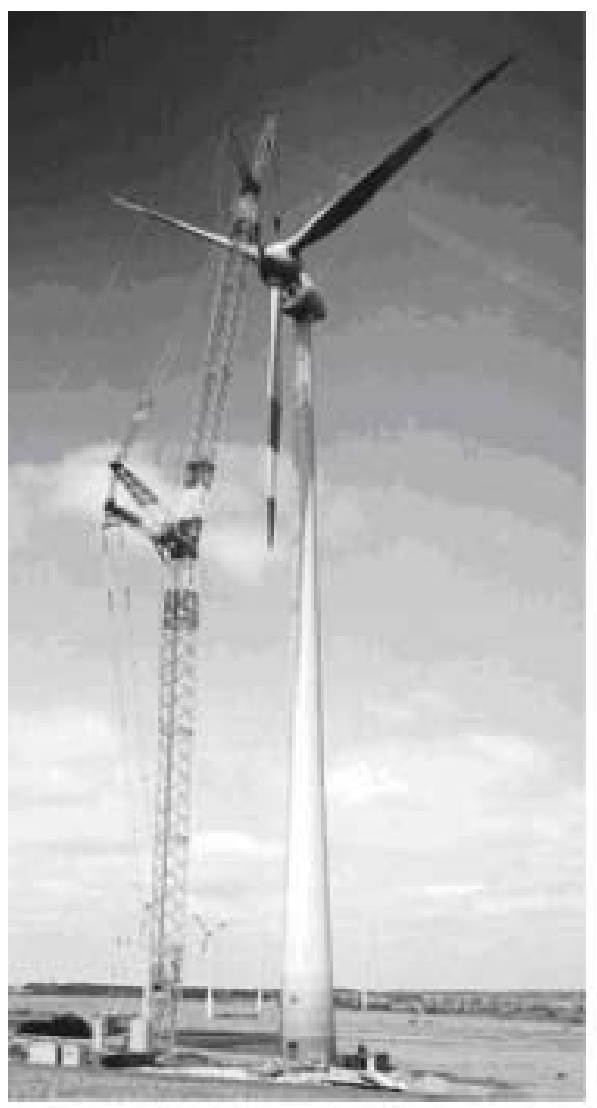

große Hubhöhe

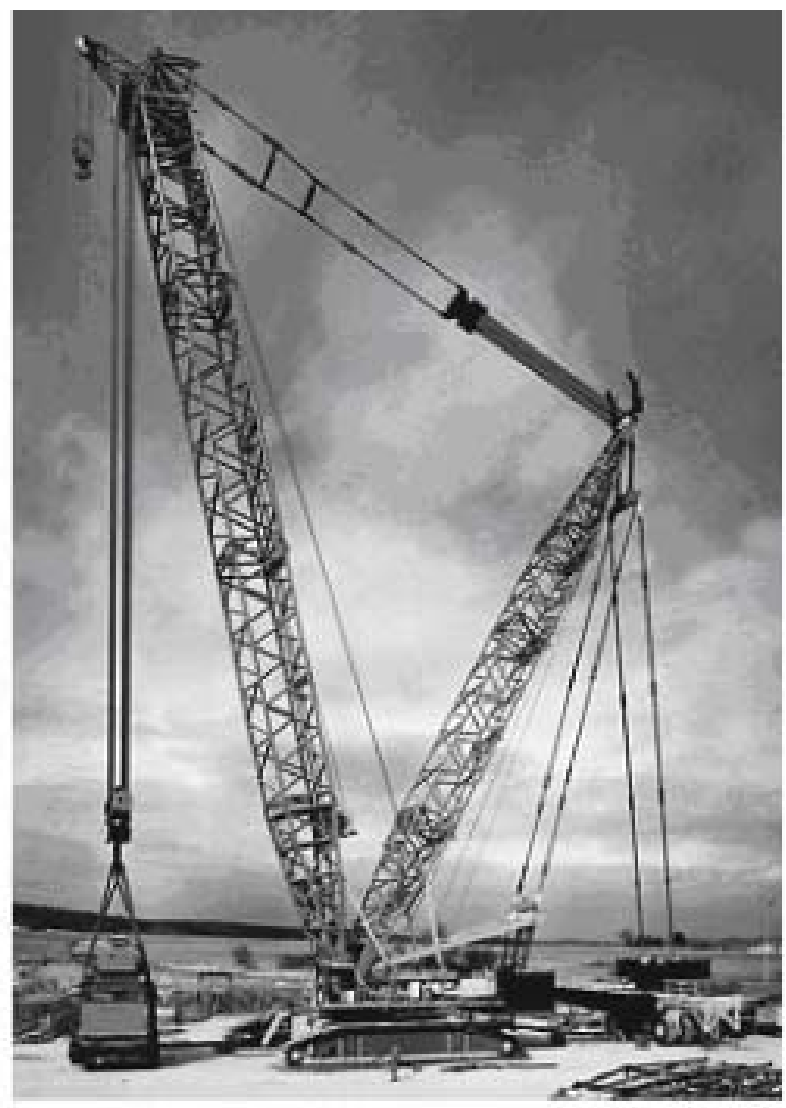

große Hublast

Abbildung 1: Hubaufgaben mit unterschiedlichen Anforderungen

Dieses besteht, neben Ober- und Unterwagen, aus einer begrenzten Zahl von Gittermastbauteilen für das Auslegersystem. Entsprechend der Variationsmöglichkeiten der einzelnen Auslegertypen lässt sich daraus eine Vielzahl unterschiedlicher Ausrüstungsvarianten zusammenstellen. Abbildung 2 zeigt drei Beispiele von durchschnittlich vierzig Kombinationsmöglichkeiten eines Geräts [Dömök95].

Jede Ausrüstungsvariante kann zudem in verschiedenen Auslegerlängen (Rüstzustände) aufgebaut werden. Allein durch die unterschiedlichen Längen können sich für eine Ausrüstungsvariante bereits bis zu siebzig verschiedene Rüstzustände ergeben. Nicht jede Ausrüstungsvariante kann in so vielen verschiedenen Kombinationen aufgebaut werden, jedoch ergeben sich für einen Gittermast-Fahrzeugkran oft bis zu fünfhundert verschiedene Rüstzustände.

Entsprechend der Hubaufgabe und den äußeren Gegebenheiten stellt der Kranbetreiber das Auslegersystem zusammen. Als wichtigstes Hilfsmittel für die richtige Auswahl des Rüstzustandes stehen die Traglasttabellen zur Verfügung. 


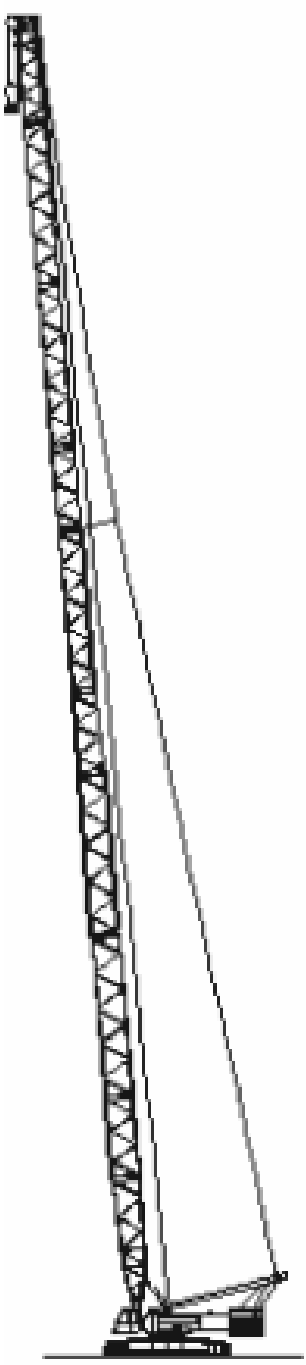

Nur Hauptausleger

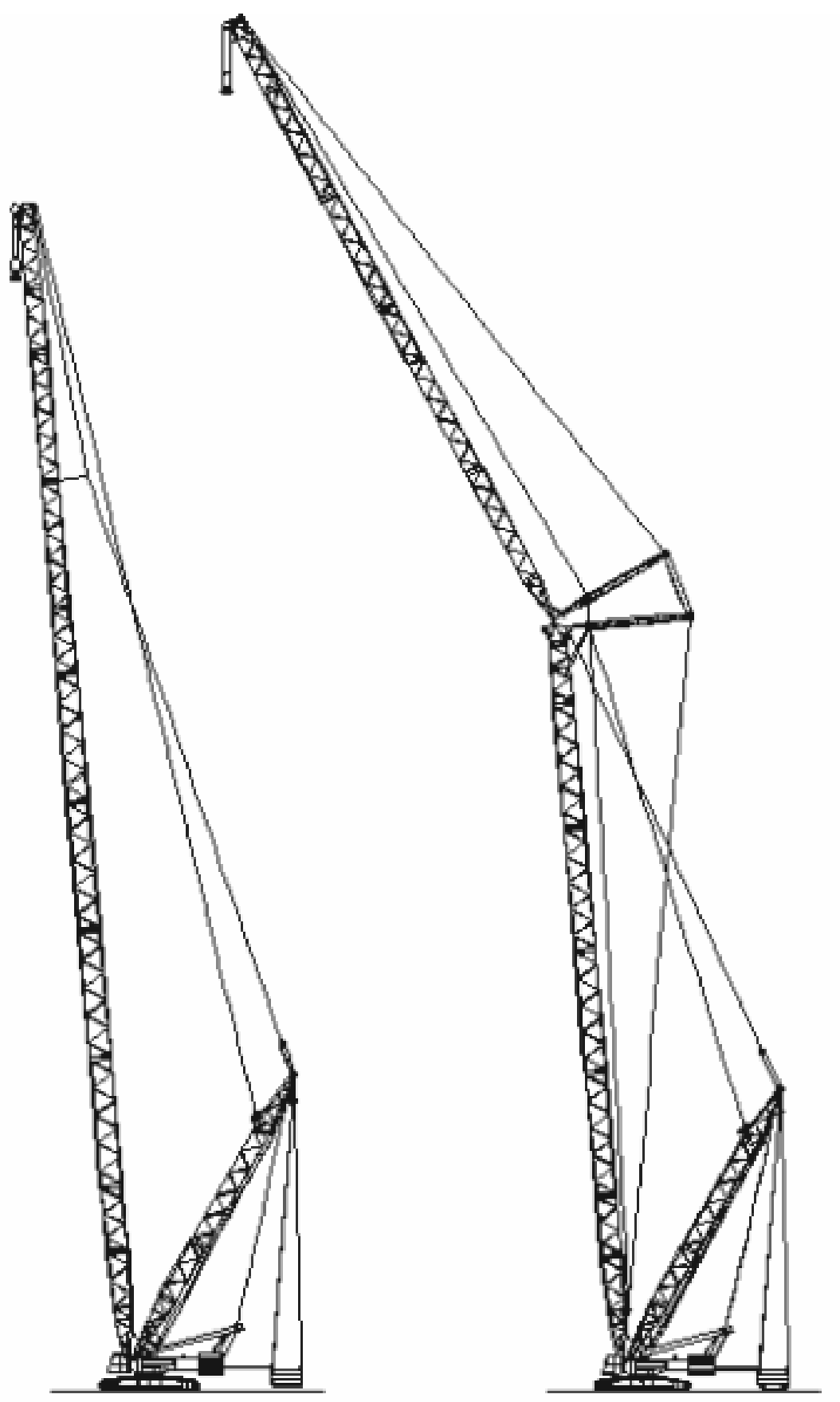

Hauptausleger mit Superlift
Haupt- und Hilfsausleger mit Superlift

Abbildung 2: Ausrüstungsvarianten eines Gittermast-Fahrzeugkrans

Eine Traglasttabelle enthält in tabellarischer Form eine Traglastkurve, die für jeden Rüstzustand (Ausrüstungsvariante in einer bestimmten Ausbaulänge) die maximale Hublast in Abhängigkeit der Auslegerstellung (Ausladung) beschreibt. Die Erstellung der Traglastkurven gehört neben der Optimierung der maximalen Tragfähigkeit zu den Hauptaufgaben bei der Berechnung eines Fahrzeugkrans. Dabei ist heute der Einsatz der Finite-Elemente-Methode (FEM) Stand der Technik. Sie ermöglicht, bei der Konzipierung dieser Geräte eine optimale Lösung für das Verhältnis von Traglast zum Eigengewicht zu finden.

Auch wenn in der Praxis nicht jede denkbare Stellung des Systems berechnet wird, ist für jeden Rüstzustand eine Mindestanzahl an Traglastpunkten zu ermitteln. Ein einzelner Traglastpunkt gibt für eine definierte Auslegerstellung die maximale Hublast an, für die alle geforderten Nachweise nach den gültigen Normen erfüllt sind. Alle Nachweise sind für eine Reihe verschiedener Lastfälle zu führen. Diese erfassen spezielle Beanspruchungen, besondere Betriebsweisen sowie äußere Gegebenheiten während des Einsatzes von Fahrzeugkranen. Zusätzlich sind bei der Bestimmung von Traglastpunkten noch weitere Parameter zu berücksichtigen. 
Dies sind zum Beispiel der maximal zulässige Bodendruck des Raupenfahrwerks oder die maximal übertragbare Kraft der Rollendrehverbindung.

Die Anzahl der durchzuführenden Berechnungen ergibt sich aus der Vielfalt möglicher Rüstzustände, der verschiedenen Auslegerstellungen, der notwendigen Traglastpunkte einer Traglastkurve und schließlich aus der Nachrechnung aller Lastfälle nach Norm. Mehrere Hunderttausend Rechenläufe sind deshalb bei Fahrzeugkranen keine Seltenheit. Da Gittermast-Fahrzeugkrane nur in kleinen Stückzahlen und häufig nach speziellen Kundenwünschen gefertigt werden, verursachen die Konstruktion und die Berechnung bei der Auftragsabwicklung einen hohen Zeit- und Kostenaufwand.

Da der prinzipielle Ablauf einer FE-Berechnung für jeden Rüstzustand, Stellung und Lastfall identisch ist, also algorithmischen Charakter hat, bietet sich aufgrund der großen Anzahl der notwendigen Berechnungen eine Automatisierung der Abläufe an. Dabei kommt es in besonderem Maße auf eine automatisierbare Modellgenerierung an.

Der hier vorgestellten Modellierungsmethode liegt die Abbildung von Finite-Elemente-Modellen als kinematische Strukturen zugrunde. Dabei wird das Gesamtmodell aus einer Reihe einzelner FE-Teilmodelle aufgebaut, die durch Gelenke miteinander verbunden sind. Diese Gelenkverbindungen lassen sich in Form kinematischer Bindungen mathematisch formulieren und schränken somit die Bewegungsmöglichkeit der einzelnen Glieder zueinander ein. Das Gesamtmodell wird demzufolge durch eine kinematische Kette beschrieben, in der sich die räumliche Anordnung der FE-Teilmodelle zueinander, eingeschränkt durch einen Satz von kinematischen Bindungen, in Position und Orientierung verändern kann. Die unterschiedlichen Stellungen des Systems können mit Hilfe eines einzigen Modells durch das Verändern weniger Parameter sehr einfach und zuverlässig erzeugt werden. Diese Eigenschaft ist für die Automatisierung der Berechnung derartiger Systeme von außerordentlicher Bedeutung.

Aufbauend auf den hier beschriebenen theoretischen Grundlagen wurde das Programmsystem VisualNODYA (vgl. [Günthner04]) entwickelt, das eine von der Modellierung bis hin zur Auswertung durchgängige Berechnung von Finite-Elemente-Modellen mit veränderlicher räumlicher Ausprägung ermöglicht. Bei der Anwendung von VisualNODYA in verschiedenen Berechnungsprojekte konnte gezeigt werden, dass mit der hier vorgestellten Modellierungsmethode die Effektivität des Berechnungsverfahrens gesteigert und damit verbunden die Entwicklungszeit verkürzt werden kann.

\section{Kinematische Ketten}

Maschinen bestehen in der Regel aus einer Reihe einzelner Teilkörper (Baugruppen, Bauteile), die durch Lagerungen, Führungen, Verbolzung etc. so miteinander gekoppelt sind, dass die zur Erfüllung der Funktion der Maschine in Verbindung stehenden Bewegungen und Kraftübertragungen bestmöglich erreicht werden. Um diese Wechselwirkungen zu beschreiben, bedient man sich sog. Mehrkörpersysteme (MKS), die sich auch zur Beschreibung großer Verschiebungen eignen, was der normalen Betriebsweise solcher Maschinen entspricht.

Während man mit Hilfe von Mehrkörpersystemen die Bewegungsgrößen (Geschwindigkeiten, Beschleunigungen) der Einzelkörper, die Zwangskräfte und Momente in den Bindungsstellen untersucht [Garcia94, Shabana94, Shabana98], beschränkt man sich bei kinematischen Ketten auf rein kinematische Betrachtungen. Es werden lediglich die Bewegungen des mechanischen Systems betrachtet, ohne nach den Ursachen zu fragen.

Eine kinematische Kette besteht aus einer endlichen Anzahl von Gliedern, die durch Gelenke miteinander verbunden sind. Ein Gelenk lässt Bewegungen in bestimmten Freiheitsgraden oder Relativbewegungen zwischen einzelnen Gliedern zu oder schränkt sie ein. Kinematische Ketten können als offene oder geschlossene Strukturen ausgeprägt sein (vgl. Abb. 3).

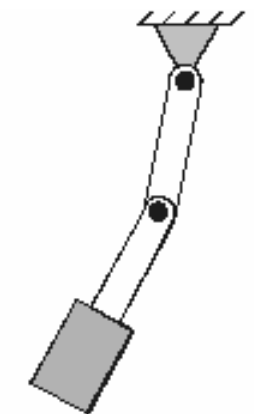

offene kinematische Kette

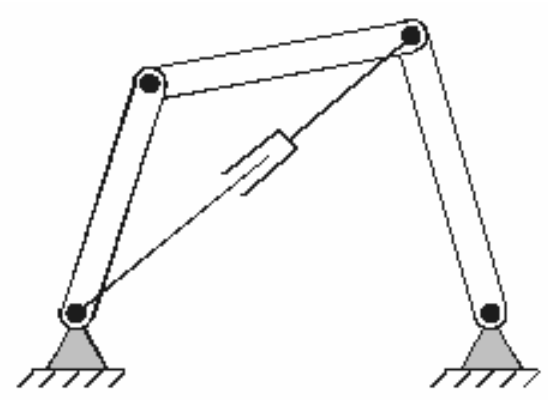

geschlossene kinematische Kette

Abbildung 3: Unterscheidung kinematischer Ketten 


\section{Modellbildung}

Mit der im folgenden Kapitel vorgestellten Modellierungsmethode können Finite-Elemente-Modelle als kinematische Strukturen abgebildet werden. Dadurch ergibt sich die Möglichkeit, auf einfache Weise durch das Ändern weniger Parameter in kurzer Zeit unterschiedliche räumliche Konfigurationen des Modells zu erzeugen, um somit die verschiedenen Betriebszustände einer Maschine effizient nachzubilden.

Dieses Konzept ist in Hinblick auf eine Automatisierung der Finite-Elemente-Berechnung solcher Systeme sehr gut geeignet. Dabei sind im Unterschied zu dem in [Schröder00] beschriebenen Verfahren zur Systemmontage sowohl offene als auch geschlossene kinematische Ketten modellierbar. Außerdem kann das Modell in Form einer Baumstruktur beliebig tief geschachtelt aufgebaut werden.

Durch die Übertragung der Bewegungsmöglichkeiten von Gelenken der realen Maschine in das Finite-ElementeModell ist eine wirklichkeitsnahe Abbildung möglich.

\subsection{Das 2-Ebenen-Modell}

Die Abbildung von Finite-Elemente-Modellen als kinematische Strukturen lässt sich anhand eines 2-EbenenModells anschaulich erklären (vgl. Abb. 4). Ausgehend von einer realen Maschine wird diese in der ersten Modellebene als Starrkörperkette betrachtet. Anschließend geht man in der zweiten Ebene auf ein FiniteElemente-Modell über.
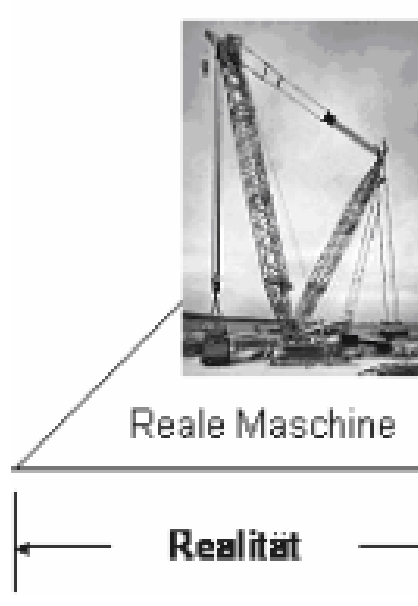

\section{Reale Maschine}

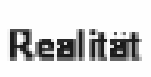

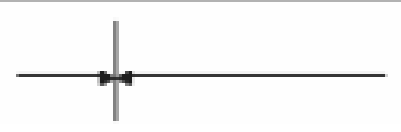

\section{Modellstufen}

Abbildung 4: 2-Ebenen-Modell

Im ersten Schritt der Modellierung wird das reale System in Baugruppen und Bauteile (Substrukturen) zerlegt. Diese stellen im Starrkörpermodell die beweglichen Glieder einer Starrkörperkette dar. Wie in [Webhofer04] gezeigt wird, trägt die Unterteilung des Gesamtsystems in Teilsysteme nicht nur zur Übersichtlichkeit des Modells bei, sondern reduziert in erheblichem Maße den Rechenaufwand bei der Lösung des Montageproblems. Unter rein kinematischer Betrachtung werden die Teilkörper gemäß den realen Lagerungen, Führungen, Verbolzungen etc. durch geeignete Bindungselemente (Scharniergelenk, Linearführung, Schiebehülse, etc.) an ihren Gelenkanschlüssen im Modell miteinander verbunden. Man erhält somit einen Mechanismus mit den Bewegungsmöglichkeiten der realen Maschine (vgl. Abb. 5). 

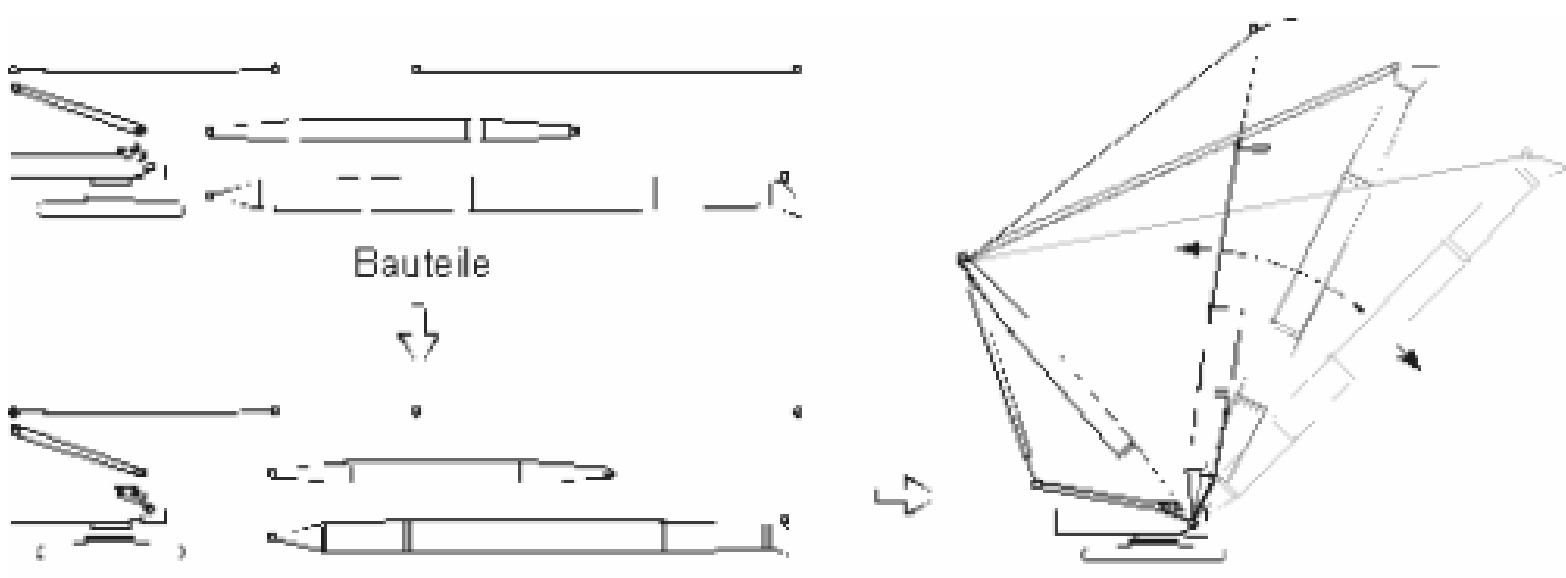

Baugruppen

\section{Starrkörpermodell}

Abbildung 5: Montage des Starrkörpermodells

In dieser Phase der Modellierung ist zur Beschreibung der einzelnen Teilkörper lediglich die räumliche Anordnung der Gelenkanschlüsse von Bedeutung. Durch Lösen des Montageproblems kann jede gewünschte Stellung der Gesamtstruktur in Abhängigkeit der Bindungsparameter (Winkel, Abstände) erzeugt werden und man erhält die Position und Orientierung jedes einzelnen Bauteils.

Wie in Abbildung 6 dargestellt, wird beim Übergang von der Starrkörper- in die FE-Modell-Ebene jedes Bauteil durch ein entsprechendes Finite-Elemente-Modell ersetzt. Die räumliche Anordnung der FE-Teilmodelle zueinander ist durch die Position und Orientierung der Bauteile gegeben.

Um aus den einzelnen FE-Teilmodellen ein Gesamtmodell zu erhalten, müssen die Teilmodelle mechanisch miteinander verknüpft werden. Die kinematischen Bindungen des Starrkörpermodells werden in Koppelbedingungen der Knotenverschiebungen umgewandelt, sodass die Beweglichkeit auch in der FE-Struktur erhalten bleibt. Dies ist nur möglich, wenn in den Verbindungsstellen entsprechende Knoten zur Verfügung stehen. Als Voraussetzung dafür sind spezielle Anschlussdefinitionen in den Bauteil-Modellen vorzunehmen. Definiert man schließlich noch Lasten und Randbedingungen, so kann am Gesamtmodell eine FE-Berechnung durchgeführt werden.

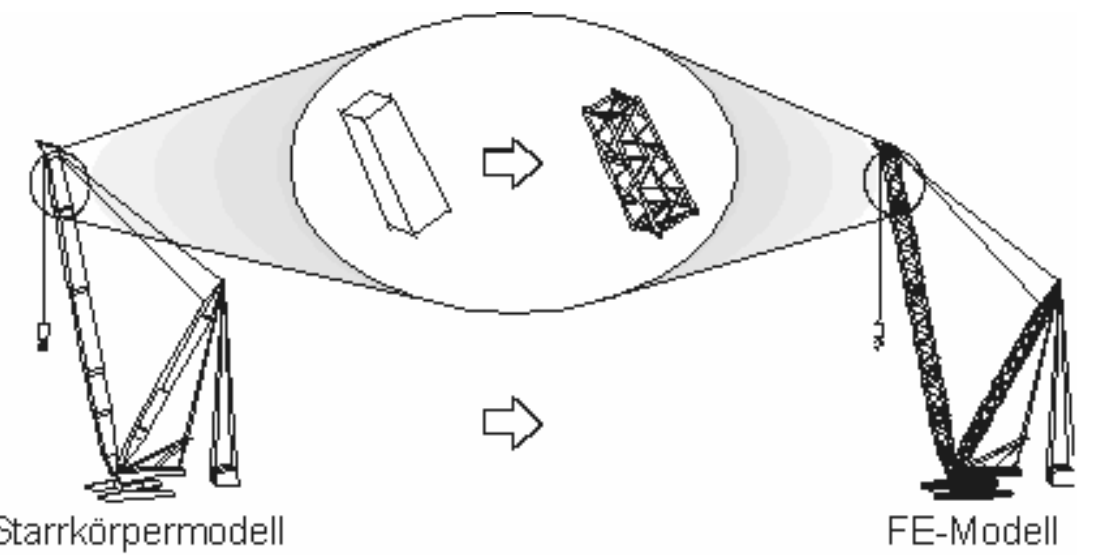

Abbildung 6: : Übergang Starrkörpermodell - FE-Modell

\subsection{Anschlüsse}

Als Anschluss $A_{i, j}$ eines Körpers ${ }{ }_{i}$ wird hier die Verbindung von ${ }^{n}$ Anschlusspunkten $P_{i, k}(k=1, \ldots, n)$ bezeichnet. Ein Anschlusspunkt kann dabei ein beliebiger Körperpunkt sein, der durch seinen Ortsvektor $\vec{p}_{i, k}$ im Bauteilkoordinatensystem ${ }^{B_{i}}$ angegeben wird (vgl. Abb. 7). 


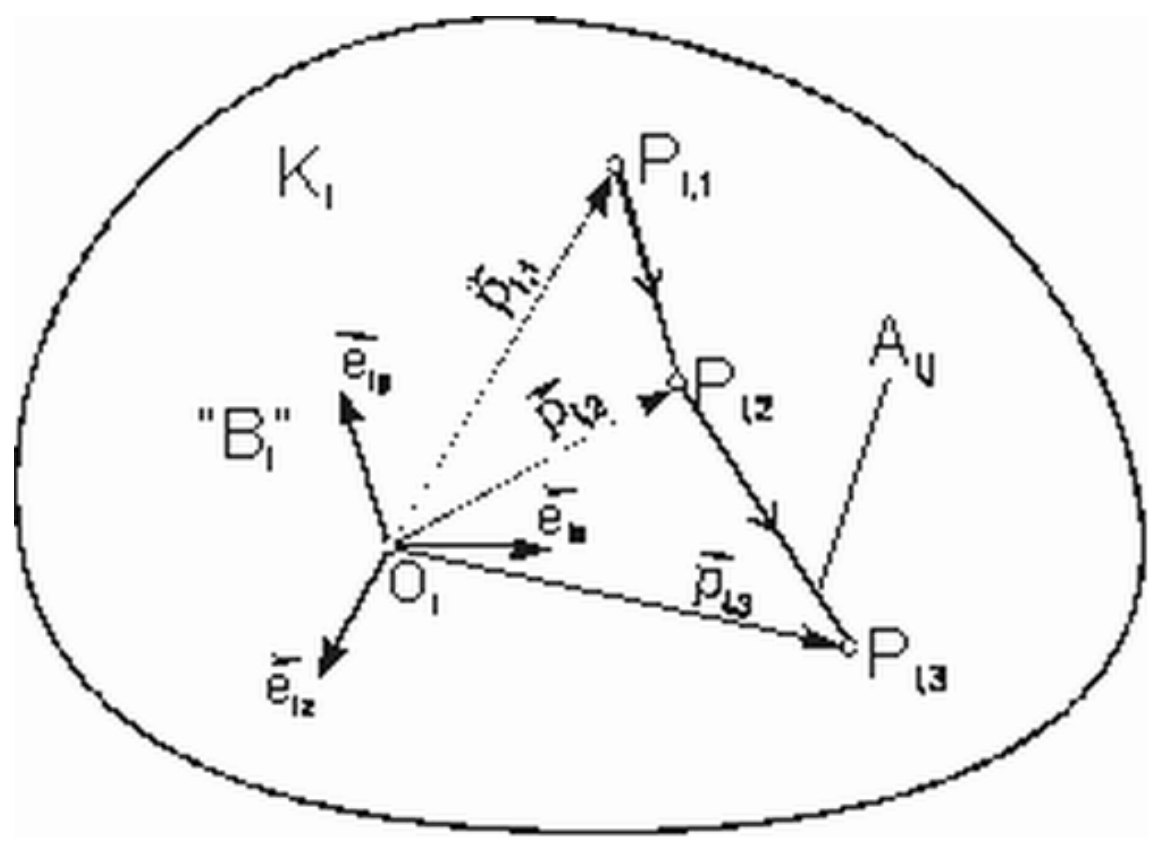

Abbildung 7: Anschlussdefinition

Die im Folgenden benötigten Anschlüsse beschränken sich auf 1-Punkt-, 2-Punkt- und 3-Punkt-Anschlüsse. Während ein 3-Punkt-Anschluss eine Ebene aufspannt, kann der 2-Punkt-Anschluss geometrisch als Gerade gedeutet werden. Der 1-Punkt-Anschluss stellt lediglich einen Raumpunkt dar.

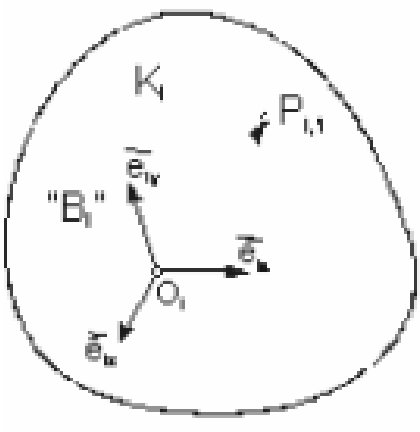

1-Punkt-Anschluss

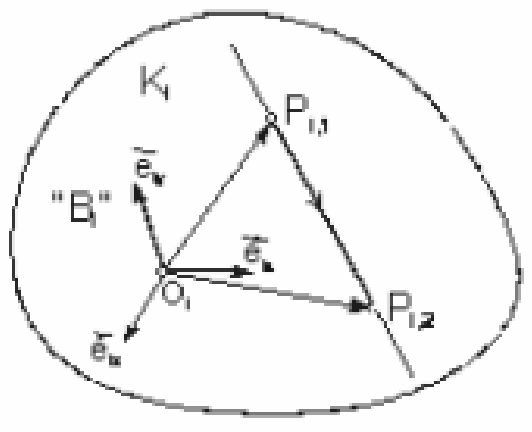

2-Punkt-Anschluss

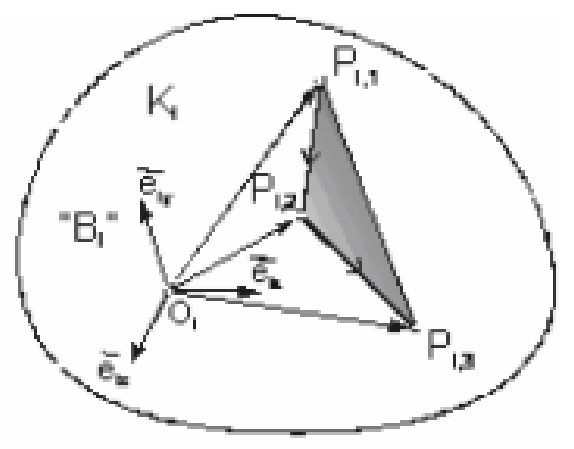

3-Punkt-Anschluss

Abbildung 8: Anschlusstypen

Bei der Strukturmontage ist lediglich die Position und Orientierung der Bauteilanschlüsse von Bedeutung. Werden zwei Bauteile durch ein Bindungselement miteinander verbunden, so wird eine bestimmte räumliche Anordnung der beteiligten Anschlüsse durch die Bindungsgleichungen erzwungen.

Möchte man beispielsweise zwei Bauteile in Form eines Kugelgelenks (vgl. Abb. 9) miteinander verbinden, so sind zur Definition zwei 1-Punkt-Anschlüsse notwendig. Die Bindungsgleichungen des Kugelgelenks würden die Anschlusspunkte $P_{1,1}$ und $P_{2,1}$ der Bauteile ${ }^{K_{1}}$ und ${ }^{K_{2}}$ stets zur Deckung bringen, so dass gilt: $\vec{v}_{1}+\vec{p}_{1,1}=\vec{v}_{2}+\vec{p}_{2,1}$ 


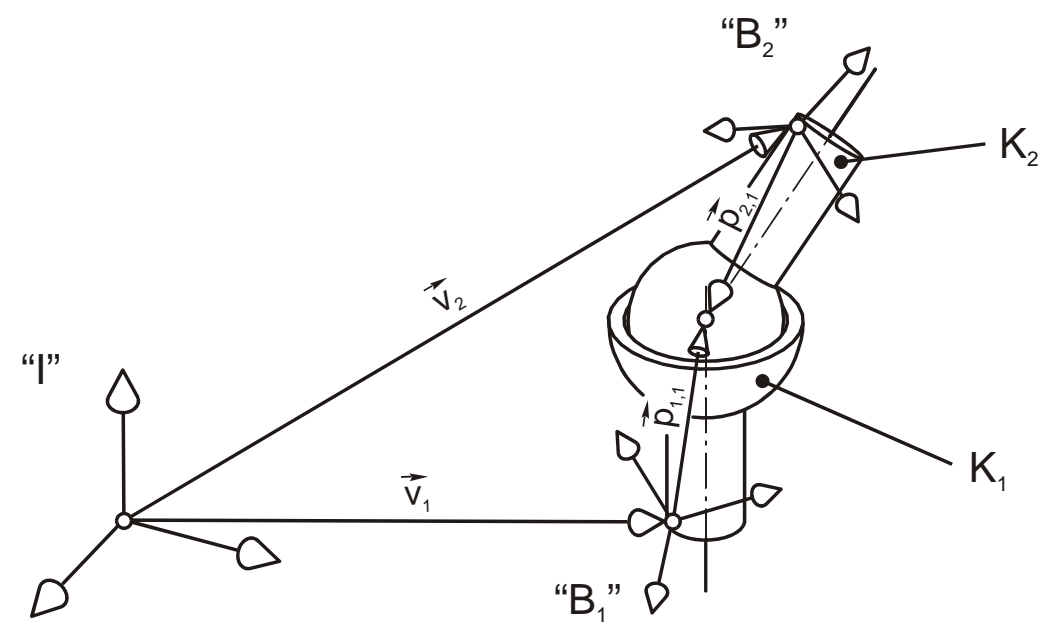

Abbildung 9: Kugelgelenk

Hinsichtlich der Verdrehung der Bauteile zueinander würde das Kugelgelenk keinerlei Einschränkungen bewirken.

Bei der Anschlussdefinition ist jedoch noch ein weiterer, wesentlicher Gesichtspunkt zu beachten. Wie bereits erwähnt, müssen nach der Strukturmontage, beim Übergang vom Starrkörpermodell zum FE-Modell, die Teilmodelle mechanisch verträglich miteinander verbunden werden. Die aus dem kinematischen Modell bekannten Bewegungsmöglichkeiten müssen auch auf die Finite-Elemente-Struktur übertragen werden, sodass die als reibungsfrei angenommenen Führungen und Lagerungen in ihren Freiheitsgraden keine Kräfte bzw. Momente übertragen.

In einem Finite-Elemente-Modell wird ein solches Gelenk mit Hilfe von Koppelbedingungen zwischen den Freiheitsgraden der Elementknoten realisiert. Als Voraussetzung muss aber gelten, dass an den Verbindungsstellen der Teilmodelle Elementknoten vorhanden sind, die sich am selben Ort befinden. Wie bereits in [Dömök95] erläutert wird, erweist sich bei der Definition von Bauteilanschlüssen die Verwendung von Elementknoten als Anschlusspunkte (vgl. Abb. 10) als sehr sinnvoll.

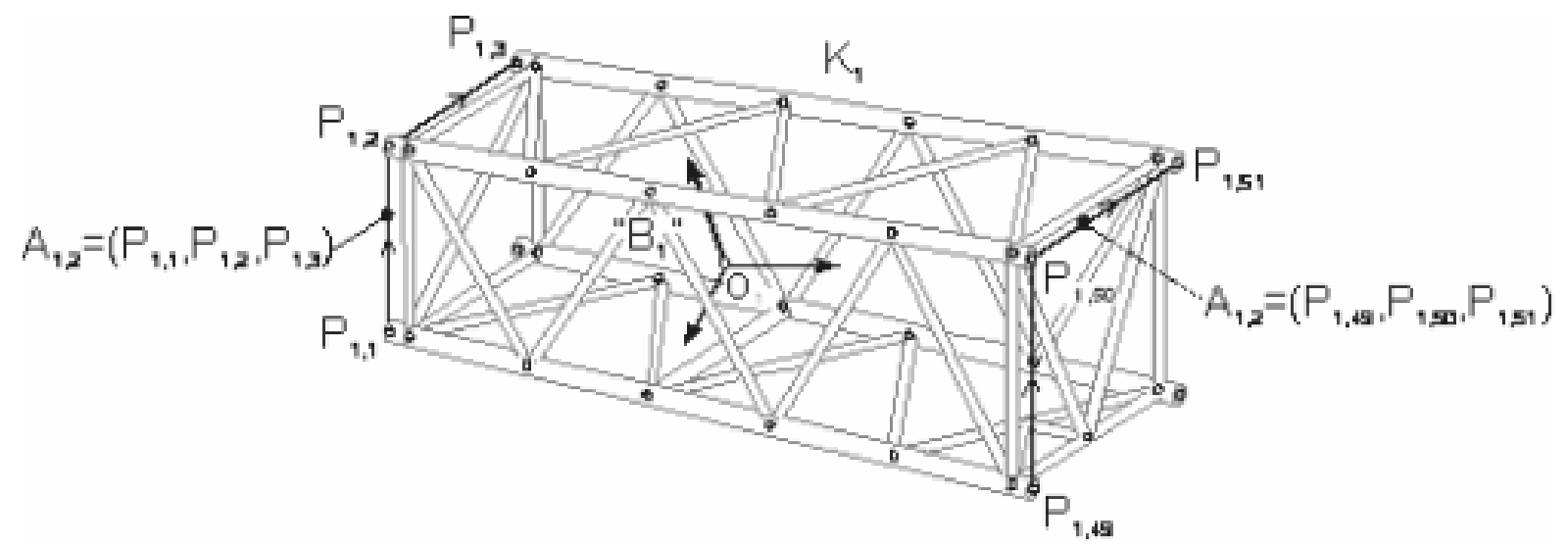

Abbildung 10: Anschlussdefinition im FE-Modell

In einem FE-Bauteilmodell wird ein Knoten durch seine Position im Bauteilkoordinatensystem eindeutig beschrieben. Verbindet man $n$ Elementknoten zu einem Anschluss, so kann dies durch spezielle Markierungselemente (tracelines) im FE-Modell geschehen. Dadurch wird klar, dass bereits bei der Modellierung der kinematischen Struktur die Teilkörper als FE-Modelle hinterlegt werden müssen, da bei der Definition der Bindungselemente die Anschlussdefinitionen benötigt werden.

\subsection{Bindungselemente}

Die Teilkörper einer kinematischen Kette können durch Gelenkverbindungen verschiedener Art miteinander verbunden werden. Dadurch werden Bewegungen in bestimmten Freiheitsgraden oder Relativbewegungen zwischen den einzelnen Gliedern zugelassen oder eingeschränkt. Die im Folgenden beschriebenen Bindungselemente gehören ausschließlich der Gruppe der holonomen Bindungen an. Somit hängen die Bindungsgleichungen lediglich von den Lagekoordinaten ab. 
Mathematisch können holonome Bindungen in fast beliebiger Ausartung beschrieben werden. In technischer Hinsicht erfüllen jedoch nur wenige Ausprägungen eine sinnvolle Funktion und sind mit den konventionellen Mitteln der Produktionstechnik herstellbar. Diese Restriktionen schränken die Anzahl theoretisch möglicher Bindungselemente stark ein. Aus den verbleibenden Möglichkeiten lässt sich eine Auswahl von Standardgelenken ableiten, die nach der Anzahl ihrer Freiheitsgrade klassifiziert werden (class I joint, class II joint, ...) können. In Tabelle 1 ist eine Auswahl von Standardgelenken dargestellt:

Tabelle 1: Übersicht Standardverbindungen

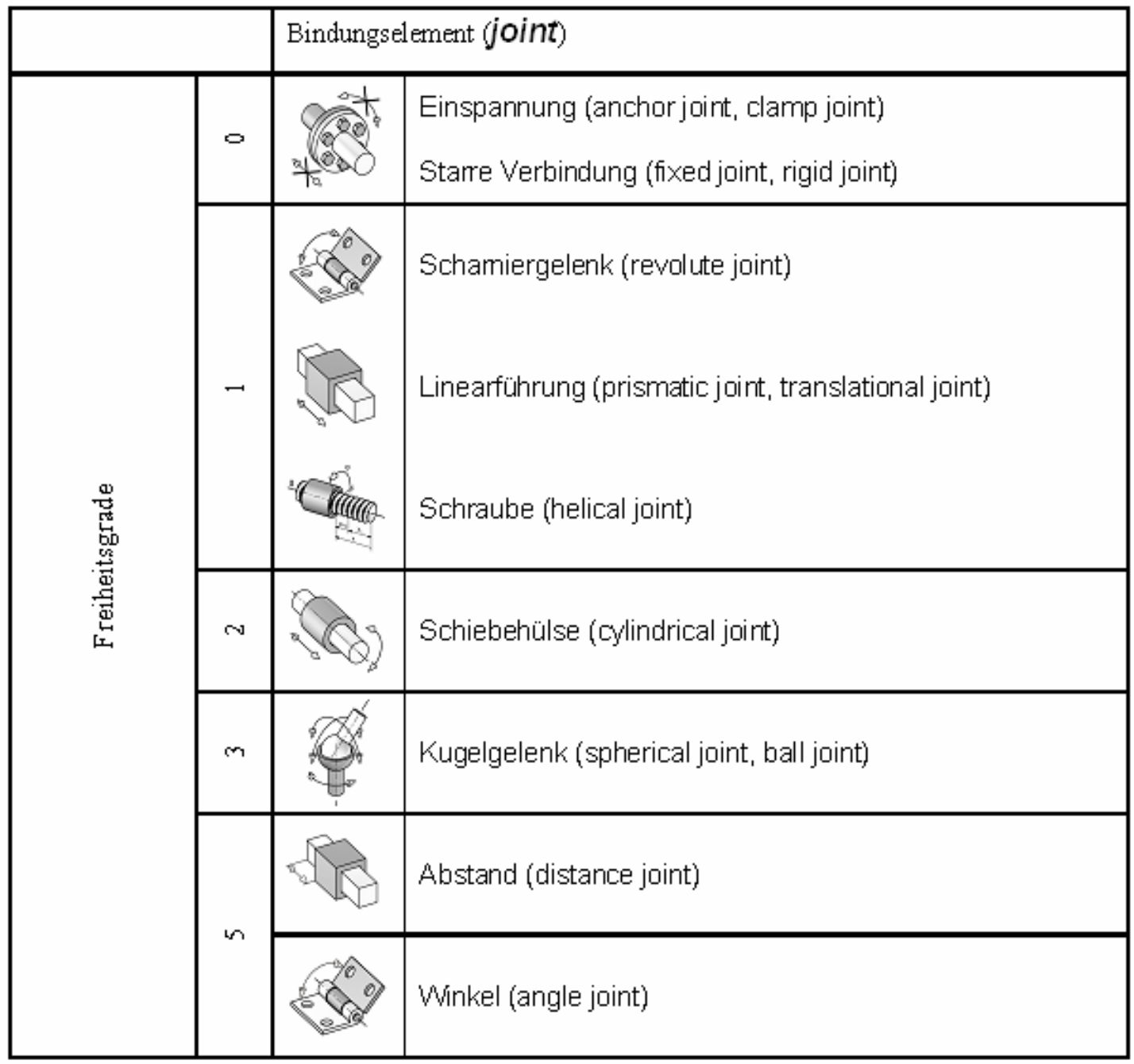

\section{Strukturmontage}

\subsection{Position und Orientierung der Teilmodelle}

Zur Beschreibung der Position und Orientierung eines Starrkörpers können Koordinatensysteme und Transformationsmatrizen benutzt werden.

Zunächst wird gemäß Abbildung 11 ein kartesisches Koordinatensystem eingeführt, welches im Anschauungsraum als eine Basis für alle darin darzustellenden Vektoren dient. Dieses Referenzsystem wird gegenüber dem Anschauungsraum als ruhend angenommen und stellt somit ein inertiales Koordinatensystem dar, das in den weiteren Betrachtungen als Inertialsystem $I\left(O_{I}, \vec{e}_{E}, \vec{e}_{Z}, \vec{e}_{E}\right)$ bezeichnet wird.

Jedem Teilkörper $K_{i}$ des Systems wird ein kartesisches Koordinatensystem $B_{i}\left(O_{i}, \vec{e}_{i x}, \vec{e}_{i y}, \vec{e}_{i z}\right)$ zugewiesen, welches in einem Punkt ${ }_{i}$ fest mit dem Körper verbunden ist. Somit kann die Position des Körpers $K_{i}$ 
gegenüber dem Inertialsystem durch die Verschiebung von ${ }^{O_{i}}$ bezüglich $O_{I}$ angegeben werden. Diese Translation wird mit dem Verschiebungsvektor ${ }_{I} \vec{v}_{i}:=\left({ }_{I} v_{i x},_{I} v_{i y},_{I} v_{i z}\right)^{T}$, gemessen in Koordinaten des Inertialsystems, ausgedrückt.

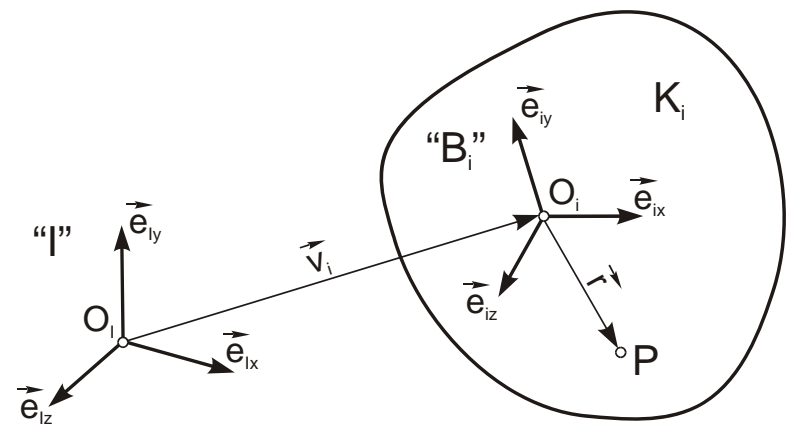

Abbildung 11: Position und Orientierung eines Starrkörpers

Zur Beschreibung der Orientierung des Körpers ${ }^{K_{\mathrm{i}}}$, gemeint ist die Verdrehung von ${ }^{B_{i}}$ gegenüber ${ }^{I}$, werden die Euler-Parameter bzw. Quaternionen verwendet. Zur Beschreibung einer beliebigen Drehung werden in beiden Fällen vier Parameter benötigt. Im Unterschied zu den Euler-Parametern sind die Quaternionen normiert. Beide beschreiben eine beliebige Drehung im Raum durch eine geeignete normierte Drehachse $\vec{u}:=\left(u_{x}, u_{y}, u_{z}\right)^{T}$ und durch einen Dreh-winkel ${ }^{\varphi}$.

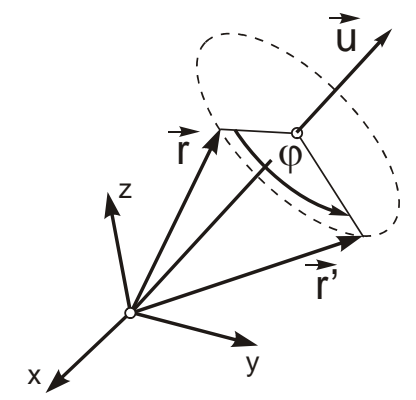

Abbildung 12: Drehung durch Drehachse und Drehwinkel

Abbildung 12 zeigt die Drehung eines Vektors $\vec{r}$ um die Achse $\vec{u}$ in die Endposition $\vec{r}^{\prime}$. Dabei zählt die Verdrehung um ${ }^{\varphi}$ im Uhrzeigersinn positiv.

Die Quaternionen $\left(e_{0}, e_{1}, e_{2}, e_{3}\right)$ sind wie folgt definiert:

$e_{0}:=\sin \frac{\varphi}{2}$

$\vec{e}:=\left(\begin{array}{l}e_{1} \\ e_{2} \\ e_{3}\end{array}\right)=\vec{u} \cos \frac{\varphi}{2} \quad b z w . \quad e_{2}=u_{x} \cos \frac{\varphi}{2}, \quad e_{2}=u_{y} \cos \frac{\varphi}{2}, \quad e_{3}=u_{z} \cos \frac{\varphi}{2}$

Die Parameter ${ }^{e_{1}}, e_{2}$ und ${ }^{e_{3}}$ geben somit die Drehachse und ${ }^{e_{0}}$ den Drehwinkel an.

Zusätzlich beschreibt folgender Zusammenhang die Abhängigkeit der Quaternionen untereinander. Dabei wird sichergestellt, dass die Drehung ohne Verzerrung erfolgt.

$e_{0}^{2}+e_{1}^{2}+e_{2}^{2}+e_{3}^{2}=1$ 
Mit Hilfe der Quaternionen lassen sich die Vektoren $\vec{r}$ und $\vec{r}^{\prime}$ aus Abbildung 12 durch

$\vec{r}^{\prime}=\underline{\underline{T}}_{Q \text { uat }} \cdot \vec{r}$

mit der orthonormalen $3 \times 3$-Drehmatrix

${\stackrel{T}{=} \rho_{u a t}}\left(\begin{array}{lll}2 e_{0}^{2}+2 e_{1}^{2}-1 & 2 e_{1} e_{2}-2 e_{0} e_{3} & 2 e_{1} e_{3}+2 e_{0} e_{2} \\ 2 e_{1} e_{2}+2 e_{0} e_{3} & 2 e_{0}^{2}+2 e_{2}^{2}-1 & 2 e_{2} e_{3}-2 e_{0} e_{1} \\ 2 e_{1} e_{3}-2 e_{0} e_{2} & 2 e_{2} e_{3}+2 e_{0} e_{1} & 2 e_{0}^{2}+2 e_{3}^{2}-1\end{array}\right)$

ineinander überführen.

Die Position und Orientierung eines Körpers ${ }^{K_{i}}$ im Inertialsystem ${ }^{I}$ kann somit durch den Vektor $\vec{x}_{i}=\left(v_{i x}, v_{i y}, v_{i z}, e_{i 0}, e_{i 1}, e_{i 2}, e_{i 3}\right)^{T}$

eindeutig festgelegt werden. Angemerkt sei an dieser Stelle, dass die sechs Freiheitsgrade eines Starrkörpers durch die sieben Komponenten von (4) beschrieben werden. Da die Quaternionen durch (2) in Beziehung zueinander stehen, bildet $\left(v_{i x}, v_{i y}, v_{i z}, e_{i 0}, e_{i 1}, e_{i 2}, e_{i 3}\right)^{T}$ kein System aus Minimalkoordinaten.

\subsection{Mathematischer Ansatz}

Im Folgenden wird ein Starrkörpersystem betrachtet, das aus ${ }^{n}$ Körpern $K_{i} i=1, \ldots, n$ besteht. Die Position und Orientierung eines Teilkörpers ${ }^{K_{i}}$ wird gemäß (4) durch die sieben Komponenten des Vektors $\vec{x}_{i}=\left(v_{i x}, v_{i y}, v_{i x}, e_{i 0}, e_{i 1}, e_{i 2}, e_{i 3}\right)^{T}$ beschrieben. Zur Angabe der geometrischen Anordnung des

Gesamtsystems wird der Vektor

$\vec{x}:=\left(v_{1 x}, v_{1 y}, v_{1 z}, e_{10}, e_{11}, e_{12}, e_{13}, \ldots, v_{n x}, v_{n y}, v_{n z}, e_{n 0}, e_{n 1}, e_{n 2}, e_{n 3}\right)$

zusammengesetzt aus den einzelnen Vektoren der Teilkörper, verwendet. Wie in Kapitel 4.1 erläutert wird, handelt es sich hierbei nicht um einen Satz von Minimalkoordinaten, da die Quaternionen $e_{i 0}, \ldots, e_{i 3}$ eines Körpers nicht unabhängig voneinander sind.

Die Lage der Körper relativ zueinander wird durch ein System von $m$ holonomen, nichtlinearen Bindungsgleichungen $F_{j}(1 \leq j \leq m)$ beschrieben. Sie stellen den Zusammenhang der Lageparameter $v_{1 x}, \ldots, e_{n 3}$ her.

Man erhält ein nichtlineares Gleichungssystem der Form:

$\vec{F}(\vec{x})=\overrightarrow{0}$

mit:

$\vec{F}(\vec{x})=\left(\begin{array}{c}F_{1}(\vec{x}) \\ \vdots \\ F_{j}(\vec{x}) \\ \vdots \\ F_{m}(\vec{x})\end{array}\right)$ 
Zur eindeutigen Montage muss das System kinematisch bestimmt sein. Dadurch ergibt sich für den Freiheitsgrad $f$ des Systems die Forderung:

$f:=7 \cdot n-m=0$

Es sind folglich $m=7 \cdot n$ linear unabhängige Gleichungen in das Gleichungssystem (6) einzufügen.

Dabei wird dem Umstand Rechnung getragen, dass zur Definition der sechs Freiheitsgrade eines Körpers bei Verwendung von Quaternionen sieben Parameter notwendig sind. In das Gleichungssystem sind zusätzlich zu den Bindungsgleichungen die Verträglichkeitsbedingungen (2) einzufügen.

Das Bindungsgleichungssystem (6) ist, wie nichtlineare Gleichungssysteme im Allgemeinen, nur numerisch durch iterative Näherungsverfahren lösbar. Bekanntlich können nichtlineare Gleichungssysteme mehrere Nullstellen haben, wobei jede Lösung $\vec{x}$ einen Zustand (hier Stellung der kinematischen Struktur) darstellt, bei dem sämtliche Gleichungen gleichzeitig erfüllt sind. Zu welcher Lösung das Näherungsverfahren strebt, hängt im Wesentlichen von den Anfangswerten zu Beginn der Iteration ab. Somit ist es bei der Montage einer kinematischen Struktur von Vorteil, wenn bereits die Ausgangslage der einzelnen Teilkörper näherungsweise der beabsichtigten Montagestellung des Systems entspricht.

\subsection{Formulierung der Bindungsgleichungen}

Im Folgenden wird exemplarisch gezeigt, wie die Bindungsgleichungen des Kugelgelenks aus Kapitel 3.2 (vgl. Abb. 9) mathematisch formuliert werden können. Eine ausführliche Beschreibung der in Tabelle 1 aufgeführten Standardverbindungen kann in [Webhofer04] nachgelesen werden.

Mit Hilfe einer „Punkt zu Punkt“-Bedingung werden gemäß Abbildung 13 die Körperpunkte $P_{1,1}$ und $P_{2,1}$ der Körper $K_{1}$ und $K_{2}$ im Raum zur Deckung gebracht.
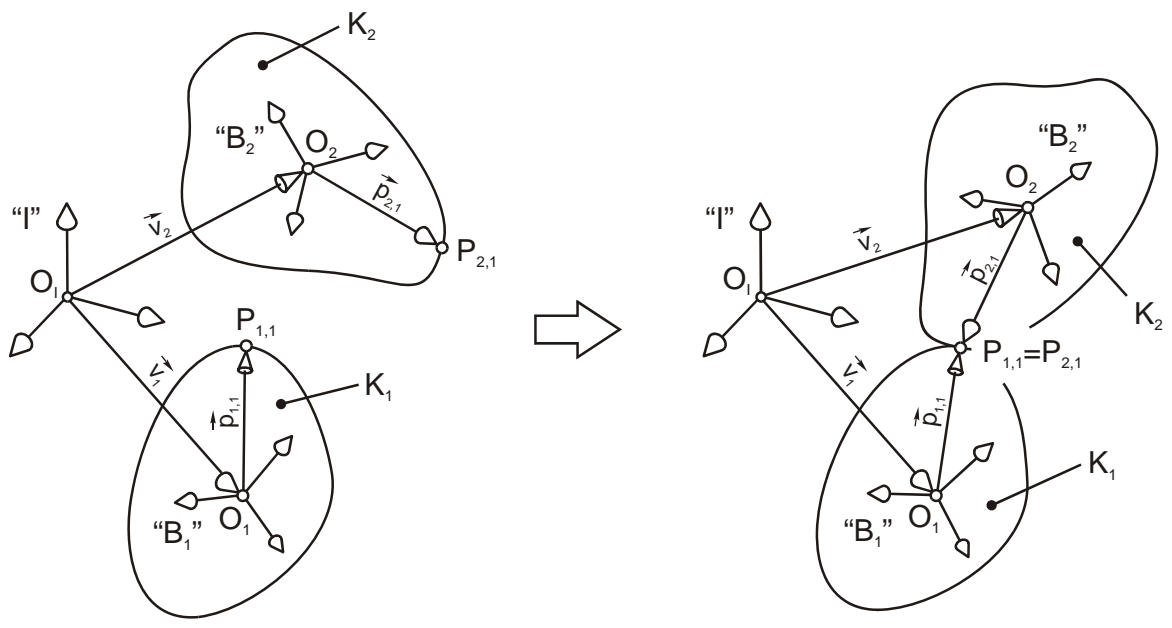

Abbildung 13: „Punkt zu Punkt“-Bedingung

Mathematisch wird dieser Zusammenhang durch die Gleichung

$\vec{F}=\vec{v}_{1}+\vec{p}_{1,1}-\vec{v}_{2}-\vec{p}_{2,1}=\overrightarrow{0}$

beschrieben. Führt man nun ein, bezüglich des Anschauungsraumes ruhendes, inertiales Bezugssystem $I\left(O_{T}, \vec{e}_{E}, \vec{e}_{z}, \vec{e}_{E}\right)$ ein (vgl. Kapitel 4.1), so schreibt man die Gleichung (8) in der Form:

$\vec{F}={ }_{I}{ }_{1} \vec{p}_{1,1}-\vec{v}_{2}-\vec{p}_{2,1}=\overrightarrow{0}$ 
Wie bereits in Kapitel 3.2 beschrieben, werden die kinematischen Bindungen des Starrkörpermodells mit Hilfe von Anschlüssen definiert. Dabei werden die Anschlusspunkte ${ }^{P_{i, k}}$ der Anschlüsse durch deren Ortsvektoren ${ }_{\xi} \vec{p}_{i, k}$ im körperfesten Koordinatensystem $B_{i}$ Hilfenahme der Transformationsvorschrift

${ }_{I} \vec{p}=\underline{T}_{\mathbb{B}_{1}} \cdot \vec{p}$

in die Form

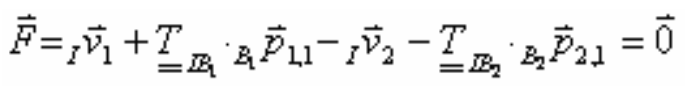

zu überführen. Dabei beschreibt $\stackrel{T}{=} \underset{\mathcal{A}}{ }$ die Verdrehung des Körpers ${ }_{i}$ im Inertialsystem $I$. Es gilt gemäß (3):

$\underline{T}_{=I B}:=\left(\begin{array}{ccc}2 e_{i 0}^{2}+2 e_{i 1}^{2}-1 & 2 e_{i 1} e_{i 2}-2 e_{i 0} e_{i 3} & 2 e_{i 1} e_{i 3}+2 e_{i 0} e_{i 2} \\ 2 e_{i 1} e_{i 2}+2 e_{i 0} e_{i 3} & 2 e_{i 0}^{2}+2 e_{i 2}^{2}-1 & 2 e_{i 2} e_{i 3}-2 e_{i 0} e_{i 1} \\ 2 e_{i 1} e_{i 3}-2 e_{i 0} e_{i 2} & 2 e_{i 2} e_{i 3}+2 e_{i 0} e_{i 1} & 2 e_{i 0}^{2}+2 e_{i 3}^{2}-1\end{array}\right)$

Verwendet man zur Beschreibung der Position und Orientierung eines Körpers den Vektor (4), dann ergeben sich aus (11) mit der Vereinfachung

$$
\left(\begin{array}{l}
\vec{T}_{i x} \\
\vec{T}_{i y} \\
\vec{T}_{i z}
\end{array}\right):=\underline{T_{I A}}
$$

die drei Komponentengleichungen ${ }^{F} \quad(j=1,2,3)_{\mathrm{zu}}$

$$
\begin{aligned}
& F_{1}={ }_{I} v_{1 x}+\vec{T}_{1 x}{ }_{B_{1}} \vec{p}_{1,1}-{ }_{I} v_{2 x}-\vec{T}_{2 x}{ }_{B_{2}} \vec{p}_{2,1}=\overrightarrow{0} \\
& F_{2}={ }_{I} v_{1 y}+\vec{T}_{1 y}{ }_{z} \vec{p}_{1,1}-{ }_{I} v_{2 y}-\vec{T}_{2 y}{ }_{Z_{2}} \vec{p}_{2,1}=\overrightarrow{0} \\
& F_{3}={ }_{I} v_{1 z}+\vec{T}_{1 z}{ }_{Z_{1}} \vec{p}_{1,1}-{ }_{I} v_{2 z}-\vec{T}_{2 z}{ }_{Z_{2}} \vec{p}_{2,1}=\overrightarrow{0}
\end{aligned}
$$

Die holonomen Bindungsgleichungen (14) bis (16) legen lediglich die Position der beiden Anschlusspunkte $P_{\mathrm{L1}}$ und $P_{2,1}$ zueinander fest. Die beiden Körper $K_{1}$ und $K_{2}$ können sich jedoch frei um den gemeinsamen Kopplungspunkt gegeneinander verdrehen, was sich anhand eines einfachen Beispiels anschaulich zeigen lässt. Würden beide Körper im Ursprung ihrer Basis $O_{1}$ und $O_{2}$ miteinander verbunden, so würden $p_{1,1}$ und $p_{2,1}$ zu $\overrightarrow{0}$

Die Bindungsgleichung (11) würde sich dann reduzieren zu:

$\vec{F}={ }_{I} \vec{v}_{1}-\vec{v}_{2}=\overrightarrow{0}$ mit $B_{1} \vec{p}_{1,1}={ }_{E_{2}} \vec{p}_{2,1}=\overrightarrow{0}$

Die Quaternionen der beiden Körper, welche die Orientierung beschreiben, kommen in (17) nicht vor und unterliegen dadurch auch keinerlei Einschränkungen. Eine „Punkt zu Punkt“-Bedingung schränkt mit ihren drei Bindungsgleichungen drei Freiheitsgrade des Systems ein. 


\subsection{Montage des Gesamtsystems}

Bei der hier vorgestellten Methode zur Abbildung von Finite-Elemente-Modellen als kinematische Strukturen können reale Systeme nach dem Baukastenprinzip aufgebaut werden. Das Gesamtsystem wird in Baugruppen und Bauteile (Substrukturen) zerlegt. Im Unterschied zu dem in [Löw93] vorgestellten Konzept kann jede Baugruppe ihrerseits weitere Baugruppen und Bauteile enthalten. Als Ergebnis entsteht eine beliebig tief geschachtelte, hierarchisch gegliederte Struktur, die baumartig durchlaufen und bearbeitet werden kann.

Wie in [Webhofer04] gezeigt wird, trägt der Aufbau des Gesamtsystems als Baumstruktur nicht nur zur Übersichtlichkeit des Modells bei, sondern reduziert in erheblichem Maße den Rechenaufwand bei der Montage. Abbildung 14 zeigt beispielhaft den hierarchischen Aufbau eines Gittermast-Fahrzeugkrans in „Hauptausleger mit Superlift“-Konfiguration, dessen Strukturbaum aus den Ebenen I, II, III und IV besteht.

In der ersten Ebene befindet sich lediglich der Wurzelknoten des Baumes, ein Platzhalter für das Gesamtmodell. Die zweite Ebene setzt sich zum Beispiel aus verschiedenen Bauteilen (Unterwagen, Oberwagen, Abspannbock etc.) und zwei Baugruppen (Superliftmast und Hauptausleger) zusammen. Die Baugruppe „Hauptausleger“ besteht ihrerseits aus fünf Bauteilen (Fußstück, Zwischenstück 6m, Zwischenstück 12m, etc.) und einer weiteren Baugruppe „Kopf“.

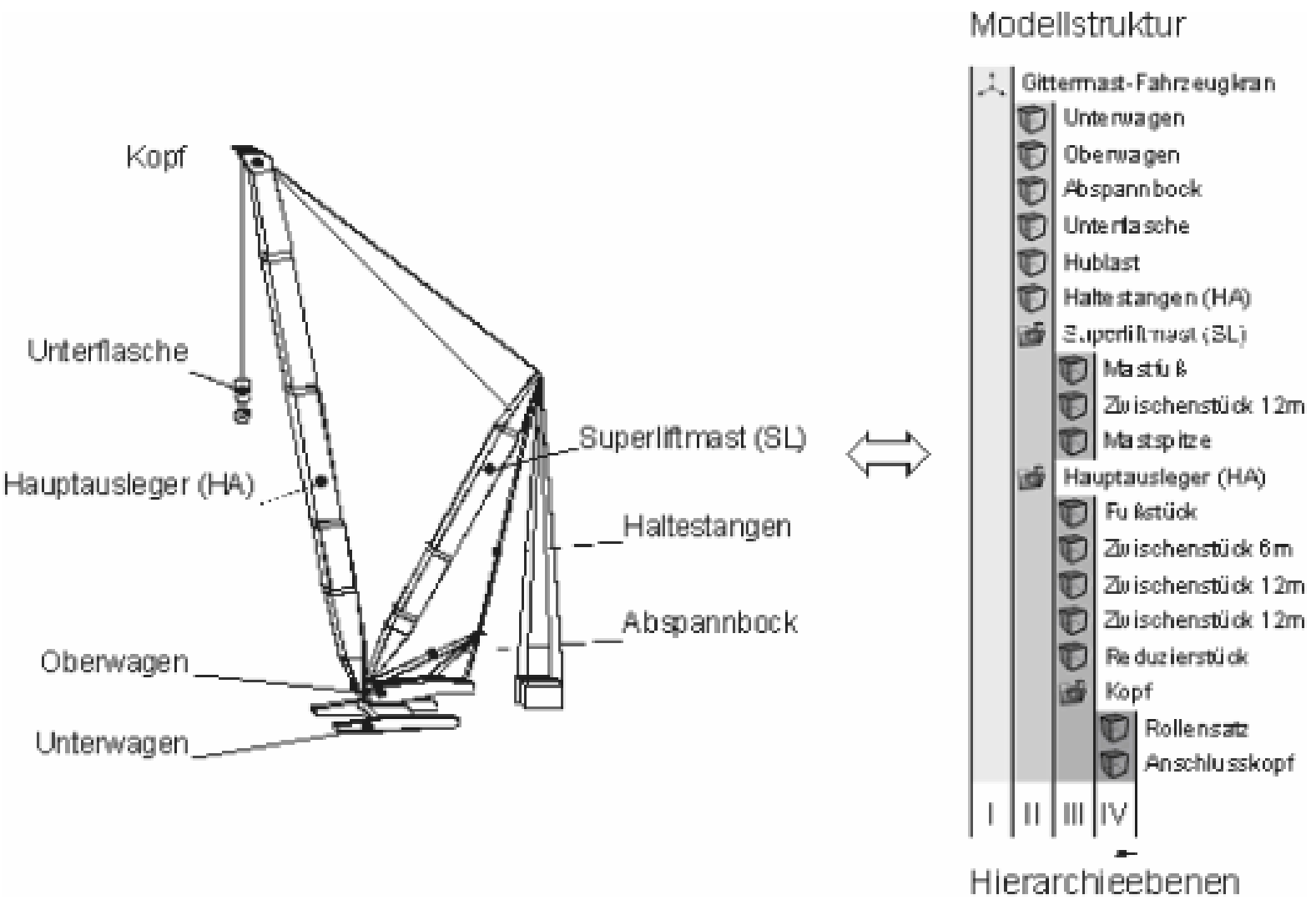

Abbildung 14: Hierarchischer Aufbau eines Gittermast-Fahrzeugkrans

Mit Hilfe des hier beschriebenen mathematischen Verfahrens lässt sich ein Starrkörpersystem, bestehend aus beliebig vielen Teilkörpern, entsprechend den kinematischen Bindungen montieren. Die Bindungsgleichungen werden dabei in Koordinaten eines inertialen Bezugssystems formuliert. Jede Baugruppe und der Wurzelknoten besitzen ein solches Bezugssystem, so dass jede Baugruppe für sich autonom montiert werden kann.

Dafür müssen folgende Voraussetzungen erfüllt sein:

- Zur Definition der Bindungselemente innerhalb einer Baugruppe können nur Anschlüsse von Bauteilen verwendet werden, die selbst Unterknoten der Baugruppe sind. Dies gilt auch für den Wurzelknoten.

- Die Substrukturen der Baugruppe müssen durch die Bindungen zu einem schlüssigen, kinematisch bestimmten System vereint werden, so dass Gleichung (7) erfüllt ist.

- Hat die zu montierende Baugruppe weitere Baugruppen als Kindknoten, so müssen diese bereits erfolgreich vormontiert worden sein, bevor diese selbst montiert werden kann. Damit wird sichergestellt, dass sich die Lage und Orientierung der zur Montage verwendeten Bauteilanschlüsse innerhalb des Kindknotens nicht mehr ändern und somit gültig sind. 
Zur Montage des Gesamtsystems wird die Baumstruktur Ebene für Ebene durchlaufen (vgl. Abb. 15). Ausgehend von der niedersten Ebene, im Beispiel aus Abbildung 14 entspricht dies der Ebene IV, wird jede Baumebene nach Baugruppenknoten durchsucht. Trifft man auf eine Baugruppe, so wird diese zusammengebaut. Erst wenn alle Baugruppen einer Ebene erfolgreich vormontiert werden konnten, kann der Montagealgorithmus versuchen, die nächst höher liegende Ebene zu montieren.

Da eine Baugruppe bei der Vormontage zwar in sich montiert werden kann, jedoch aufgrund der fehlenden Bindungen mit den Baugruppen der nächst höher liegenden Ebenen sich als Ganze im Raum frei bewegen kann, wird ein Bauteil der Baugruppe durch eine automatisch generierte Bindung im Raum festgehalten. Nach erfolgreicher Vormontage wird diese Bindung wieder entfernt.

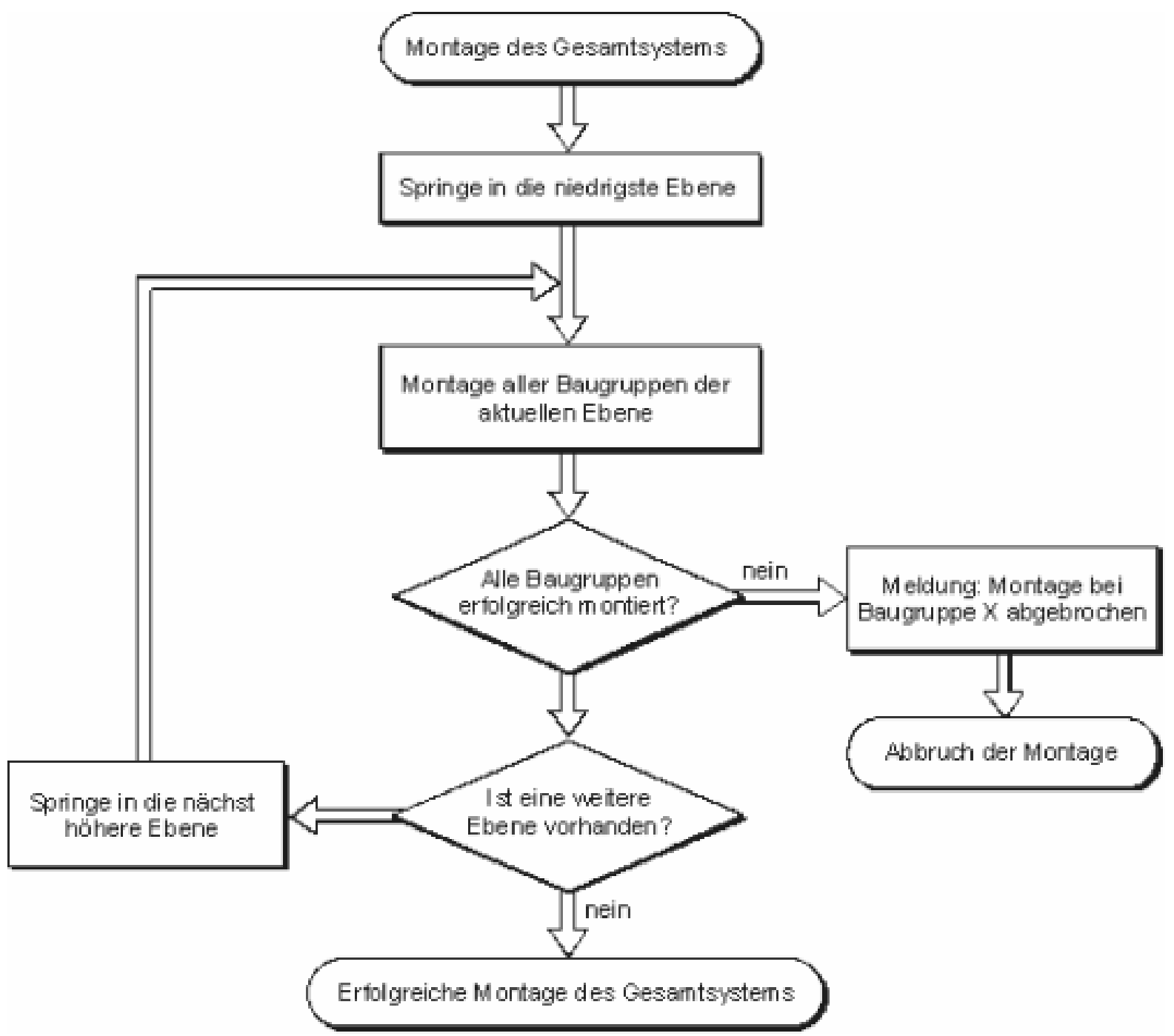

Abbildung 15: Procedere bei der Montage des Gesamtsystems

Diese schrittweise, baugruppenorientierte Montage des Gesamtsystems erweist sich auch bei fehlerhaft definierten Systemen als sehr sinnvoll, da eine unzureichend kinematisch bestimmte Baugruppe sofort identifiziert und lokalisiert werden kann.

\section{Zusammenfassung}

Mit Hilfe der Abbildung von Finite-Elemente-Modellen als kinematische Struktur ist eine effiziente FEBerechnung von Systemen, die ihre räumliche Ausprägung je nach Betriebszustand ändern, möglich. Dabei wird das Gesamtmodell aus einer Reihe einzelner FE-Teilmodelle aufgebaut, die durch Gelenke miteinander verbunden sind. Diese Gelenkverbindungen lassen sich in Form kinematischer Bindungen mathematisch formulieren und schränken somit die Bewegungsmöglichkeit der einzelnen Glieder zueinander ein. 
Das Gesamtmodell wird demzufolge durch eine kinematische Kette beschrieben, in der sich die räumliche Anordnung der FE-Teilmodelle zueinander, eingeschränkt durch einen Satz von kinematischen Bindungen, in Position und Orientierung verändern kann. Die unterschiedlichen Stellungen des Systems können durch das Verändern weniger Parameter sehr einfach und zuverlässig erzeugt werden. Diese Eigenschaft ist für die Automatisierung der Berechnung derartiger Systeme von außerordentlicher Bedeutung und weist gegenüber herkömmlichen Methoden folgende Vorteile auf:

- $\quad$ einfache Erzeugung von Stellungsvarianten durch Verändern weniger Parameter anhand eines einzigen Modells

- beliebige, sowohl offene als auch geschlossene kinematische Ketten modellierbar

- übersichtlicher und transparenter Modellaufbau durch eine Baumstruktur

- beliebig tief geschachtelter hierarchischer Aufbau der Modelle

- schrittweise, baugruppenorientierte Montage des Gesamtsystems zum einfachen Auffinden fehlerhaft definierter Teilsysteme (Baugruppen)

- Reduzierung des Rechenaufwandes bei der schrittweisen, baugruppenorientierten Montage des Gesamtsystems

- Aufwandsreduzierung bei der Modellbildung durch das Baukastensystem durch Wiederverwendung gleicher Bauteile

- große Veränderung der Gelenkparameter durch schrittweise Berechnung der Endstellung

- automatisierbare Montage für Serienberechnungen

- wirklichkeitsnahe Abbildung der Bewegungsmöglichkeiten von Gelenken der realen Maschine im Finite-Elemente-Modell

\section{Literatur}

[Dömök95]

[Garcia94]

[Günthner04]

[Löw93]

[Schröder00]

[Shabana94]

[Shabana98]

[Webhofer04]
Dömök, Stefan: Automatisierte und datenbankgestützte Berechnung von Gittermastkranen. Dissertation, Technische Universität München, 1995 García de Jalón, Javier, u.a.: Kinematic and dynamic simulation of multibody systems: the real-time challenge. Springer-Verlag, 1994

Günthner, Willibald A. u. a.: Ein flexibles Programmsystem zur Berechnung FiniterElemente-Modelle als kinematische Struktur. In: Fördern und Heben (2004) 6, S. 344-345

Löw, Hans R. E.: Automatisierung der Berechnung und Konstruktion des Stahlbaus von Fahrzeugkranen auf der Basis von FE- und CAD-Methoden. Dissertation, Technische Universität München, 1993

Schröder, Frank: Ein datenbankbasiertes Anwendersystem zur Berechnung von Gittermast-Fahrzeugkranen. Dissertation, Technische Universität München, 2000 Shabana, Ahmed A.: Computational dynamics. John Wiley \& Sons, 1994 Shabana, Ahmed A.: Dynamics of multibody systems. Cambridge University Press, 1998

Webhofer, Martin: Abbildung von Finite-Elemente-Modellen als kinematische Strukturen. Dissertation, Technische Universität München, 2004 